\title{
The Mediating Role of Spiritual Intelligence Dimensions in the Relationship between Mindfulness and Expressed Emotion in Caregivers of Epileptic Patients
}

\author{
Fahimeh Dehghani ${ }^{1}$, Faeze Ziyaei ${ }^{*}$, Neda Asemi Zavareh ${ }^{2}$
}

${ }^{1}$ Department of Psychology, Faculty of Psychology and Educational Sciences, Yazd University, Yazd, Iran

${ }^{2}$ Department of Psychology, Lorestan University, Khoramabad, Iran

\section{Article Info:}

\section{A BSTRACT}

Introduction: Caregivers' emotions can affect the quality of life of patients with epilepsy. The purpose of this study was to investigate the mediating role of dimensions of spiritual intelligence in the relationship between mindfulness and expressed emotion in the caregivers of epileptic patients. Materials and Methods: This was a descriptive cross-sectional study. The study sample consisted of 90 epileptic patients and their caregivers that were selected by convenience sampling method and responded to the expressed emotion, Kentucky Mindfulness Skills, and Spiritual Intelligence questionnaires. The hypothesis of the research was investigated by hierarchical regression analysis using the proposed steps of Baron and Kenny. Results: The hierarchical multiple regression analysis showed that in the first step, mindfulness (beta $=-0.39$ ) can predict expressed emotion. In the second step, mindfulness (beta $=-0.28$ ) and only the ability to deal with problems dimension of spiritual intelligence (beta $=-0.27$ ) predict expressed emotion. Due to the decrease of the beta value of mindfulness in the second stage compared to the first stage, the partial mediating role of the ability to deal with problems in the relationship between mindfulness and the expressed emotion is confirmed. Conclusion: Mindfulness is associated with the expressed emotion of caregivers of patients with epilepsy, and the ability to deal with problems as one of the dimensions of spiritual intelligence plays a mediating role. Therefore, it is suggested to pay more attention to psychological training in promoting the mindfulness and spiritual intelligence of caregivers of epileptic patients.

*Corresponding Author: Faeze Ziyaei

Email: f.ziaei20@yahoo.com 


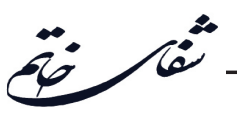

نقش واسطهاى ابعاد هوش معنوى در رابطةُ بين ذهن آتاهى و هيجان ابراز شده در مراقبين بيماران مبتلا

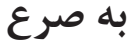

\section{فهيمه دهقانى'، فائزه ضيايى'"، ندا عاصمى زواره׳}

'اكروه روانشناسى، دانشكده روانشناسى و علوم تربيتى، دانشكاه يزد، يزد، ايران

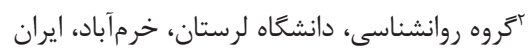

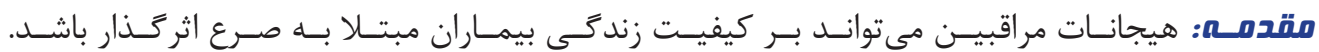

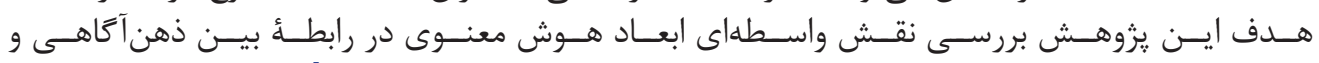

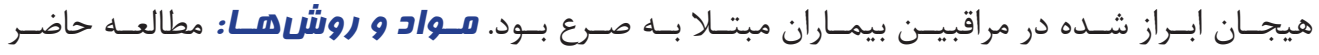

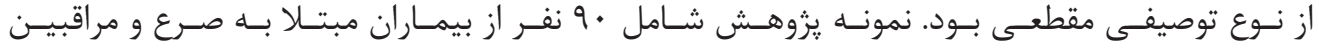

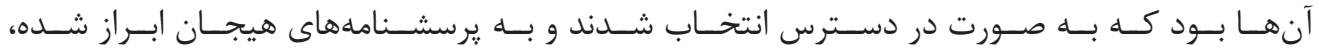

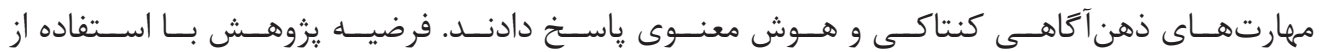

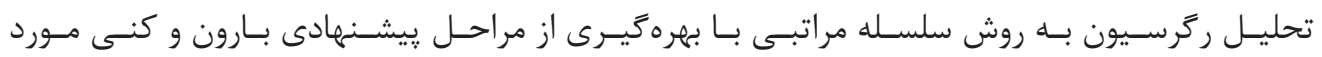

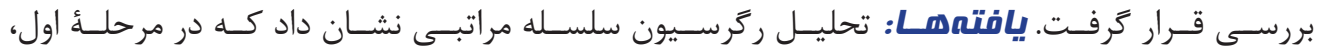

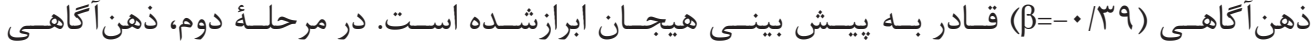

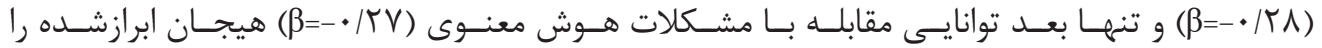

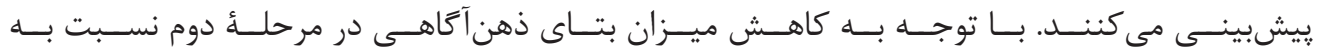

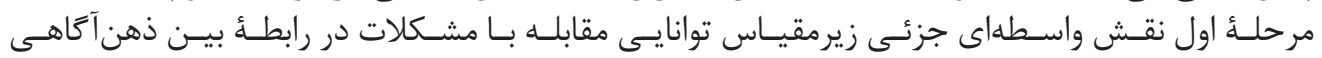

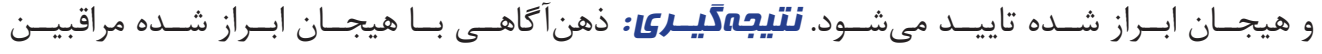

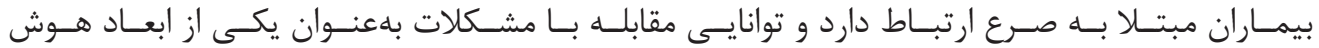

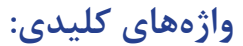

1- 1- هيجان

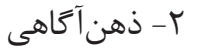

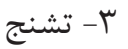
ع

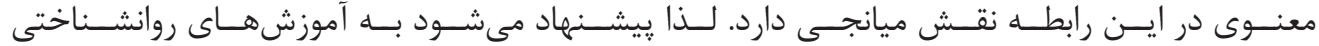

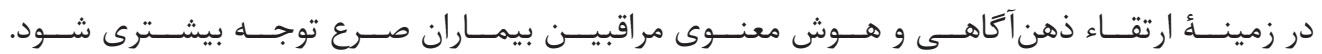




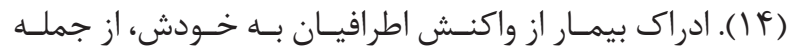

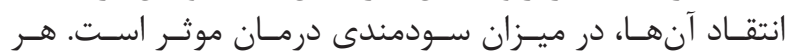

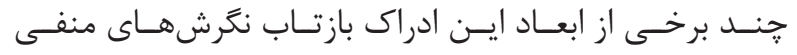

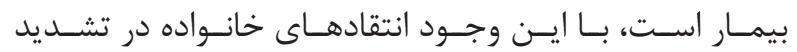

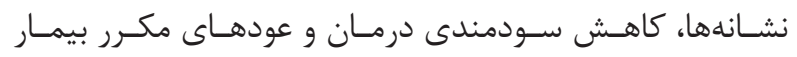

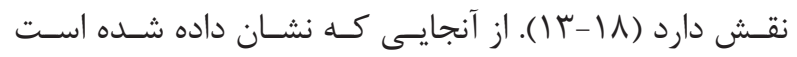

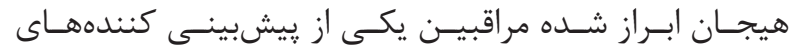

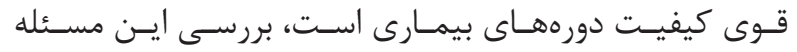

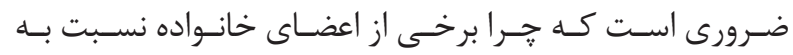

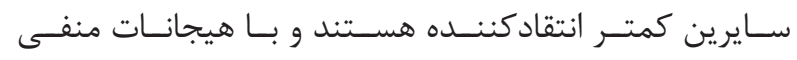

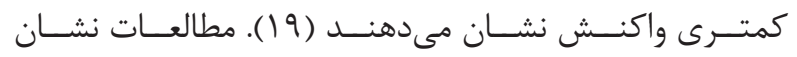

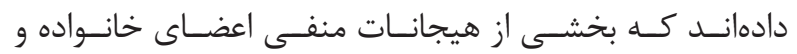

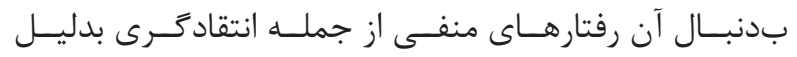

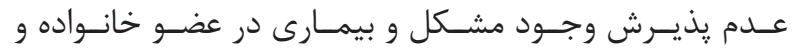

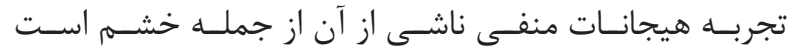

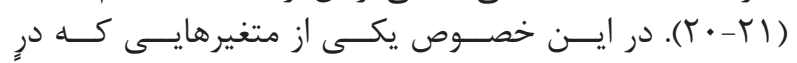

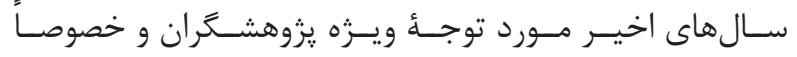

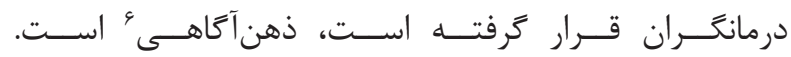

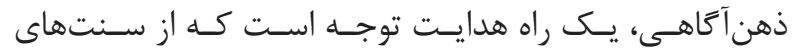

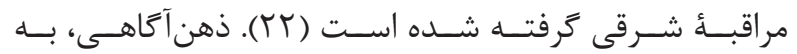

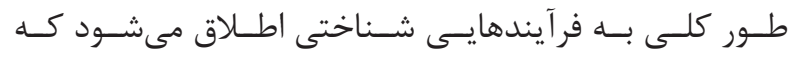

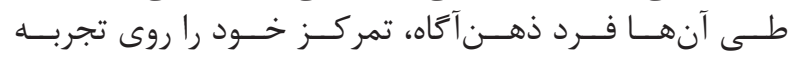

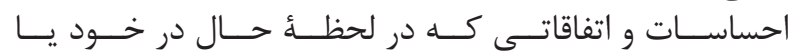

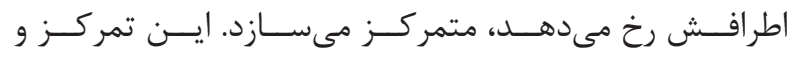

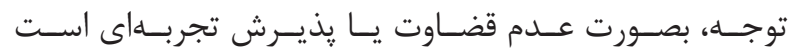

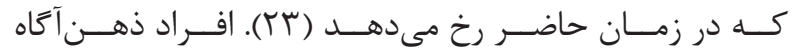

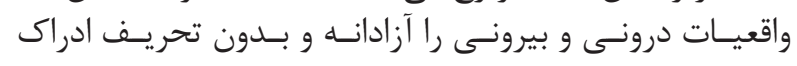

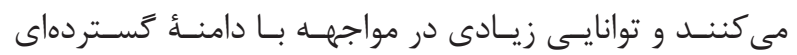

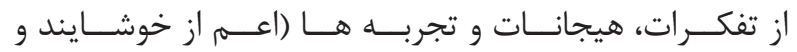

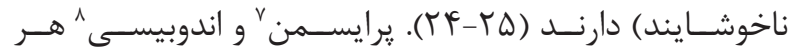

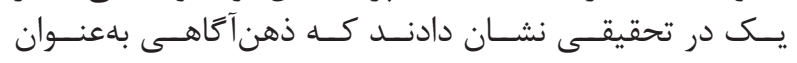

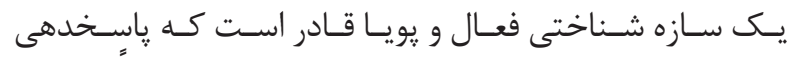

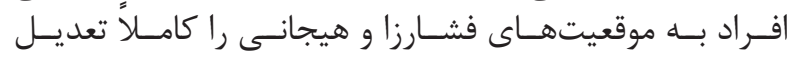

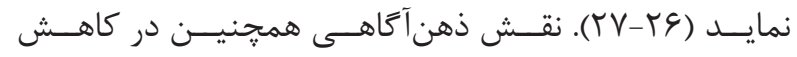

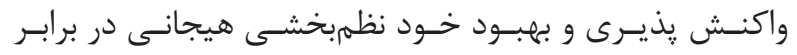

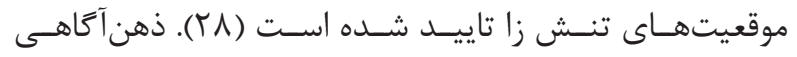

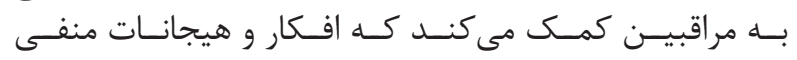

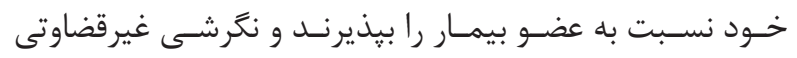

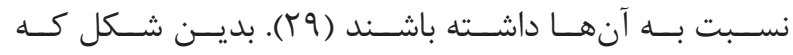

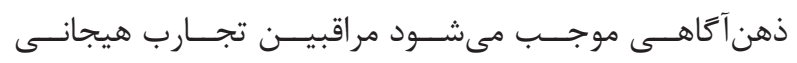

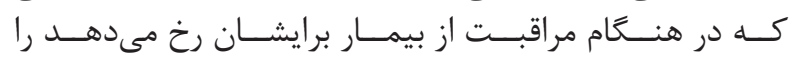

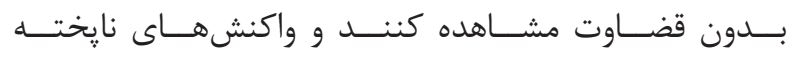

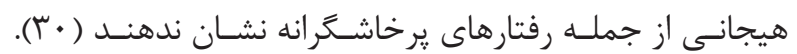

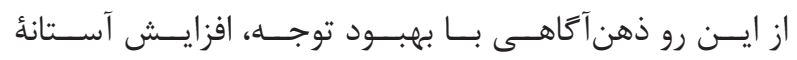

\footnotetext{
${ }^{1}$ Epilepsy

${ }^{2}$ Expressed emotion

${ }^{3}$ Criticism

${ }^{4}$ Hostility
}

\footnotetext{
${ }^{5}$ Emotional over-involvement

${ }^{6}$ Mindfulness skills

${ }^{7}$ Praissman

${ }^{8}$ Ndubisi
}

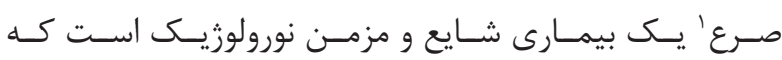

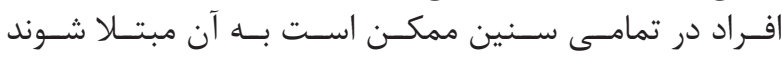

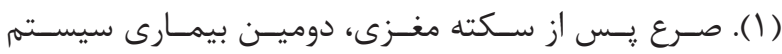

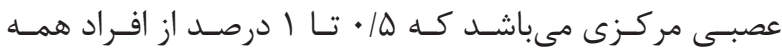

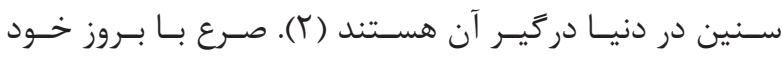

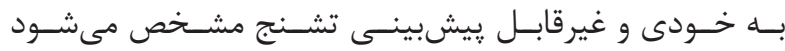

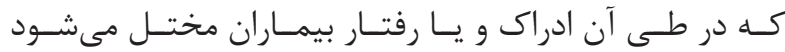

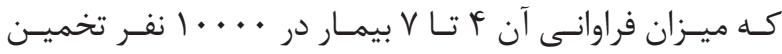

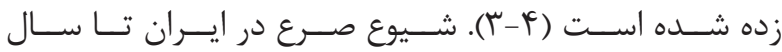

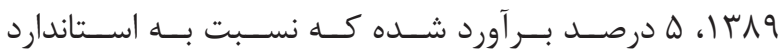

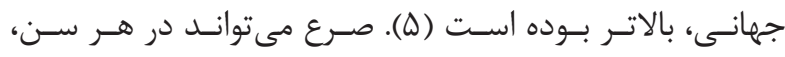

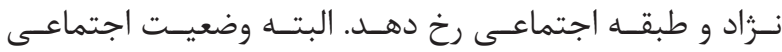

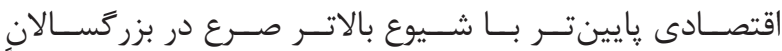

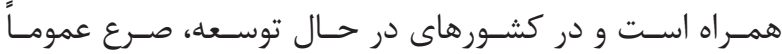

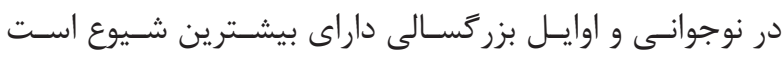

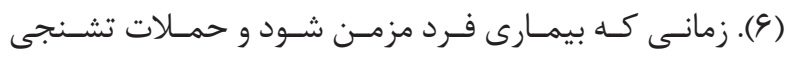

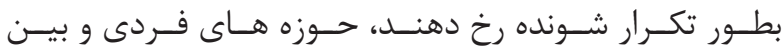

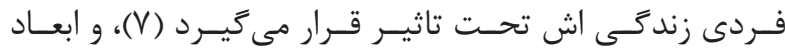

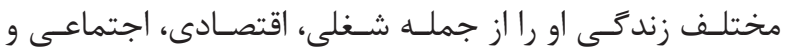

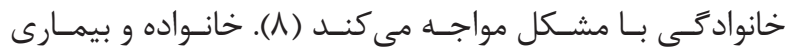

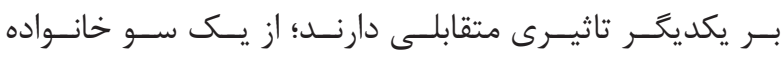

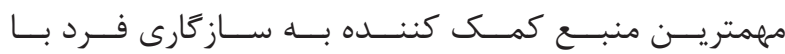

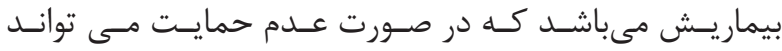

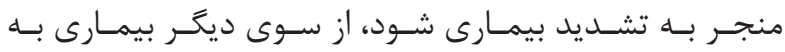

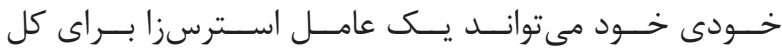

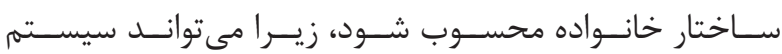

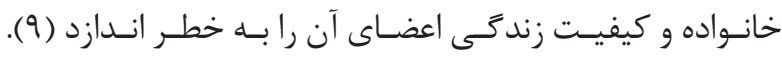

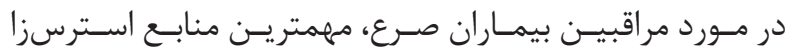

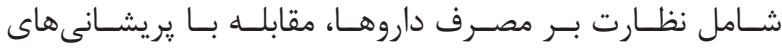

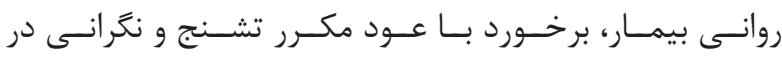

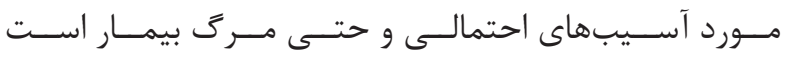

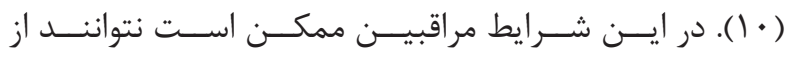

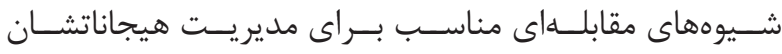

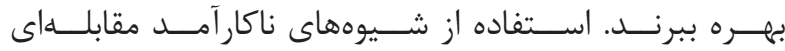

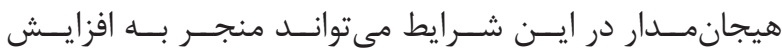

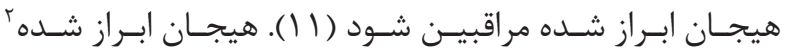

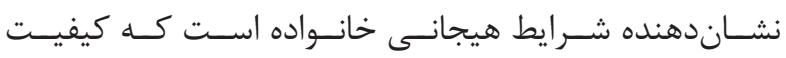

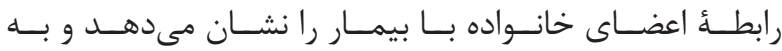

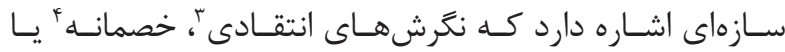

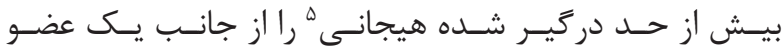

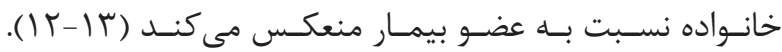

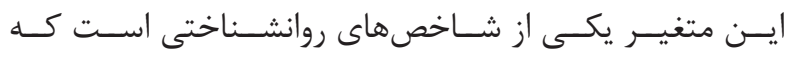

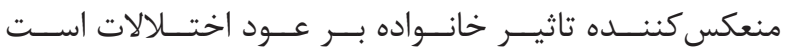




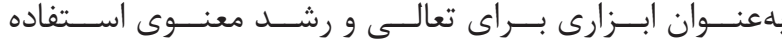

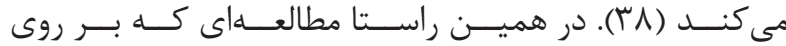

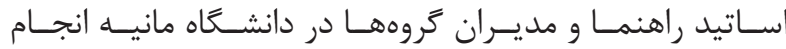

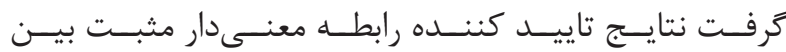

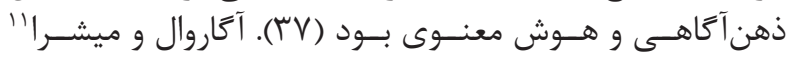

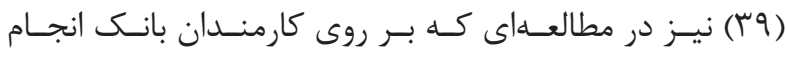

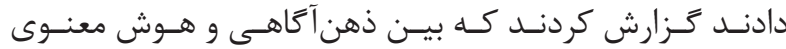

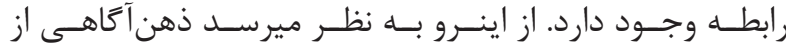

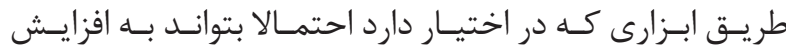

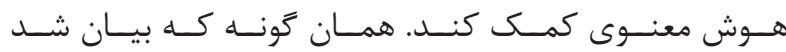

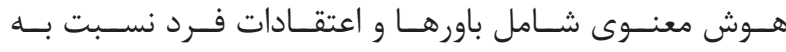

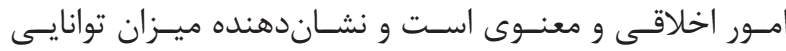

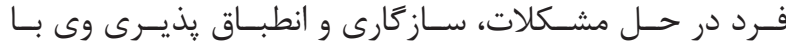

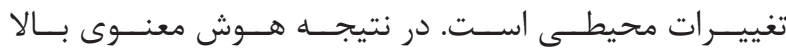

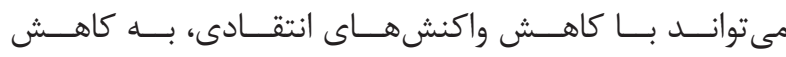

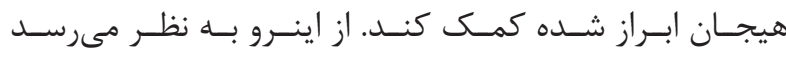

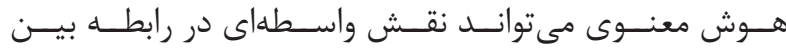

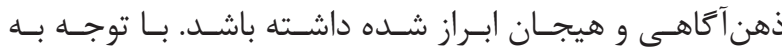

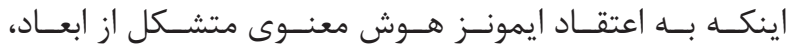

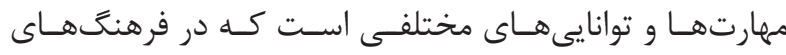

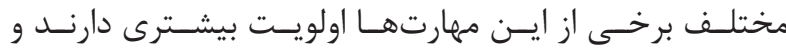

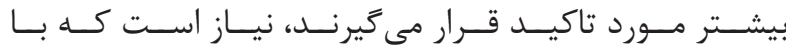

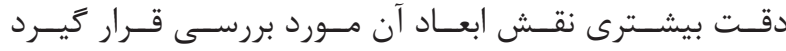

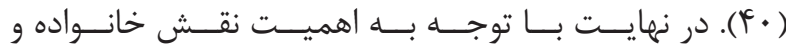

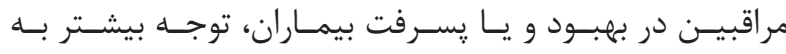

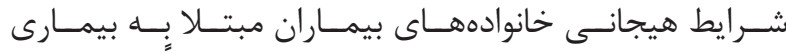

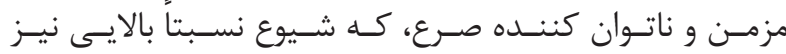

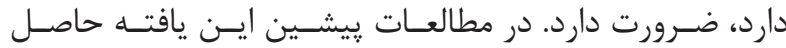

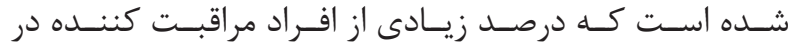

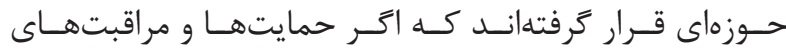

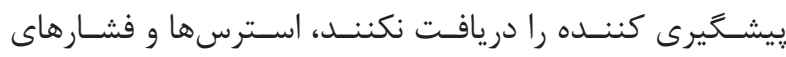

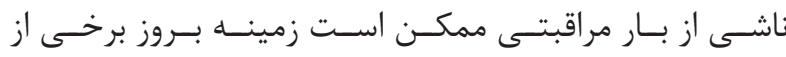

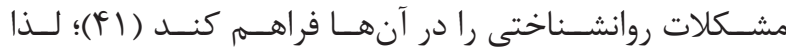

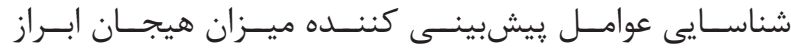

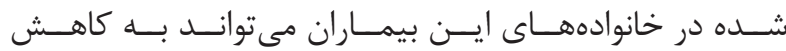

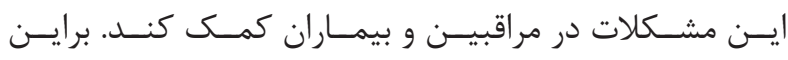

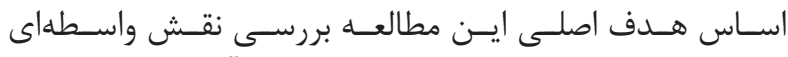

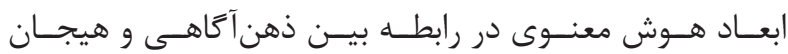

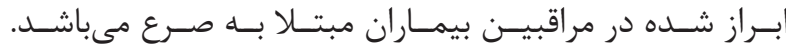

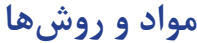

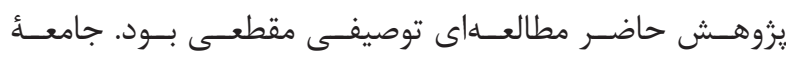

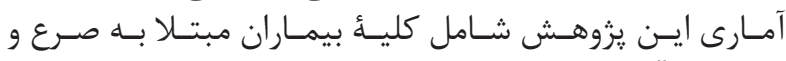

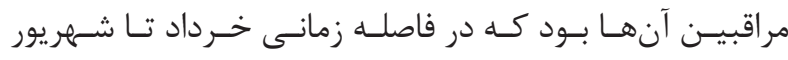

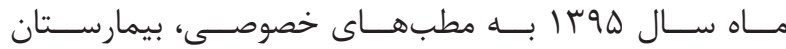

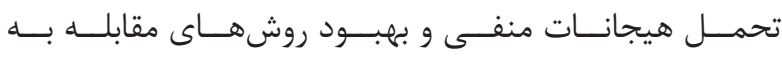

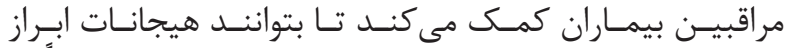

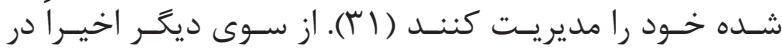

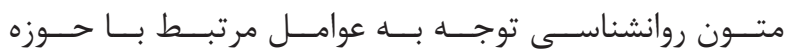

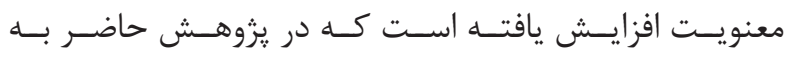

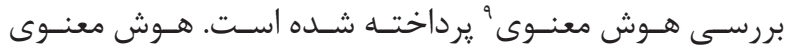

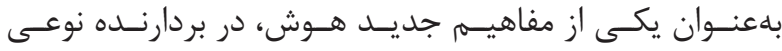

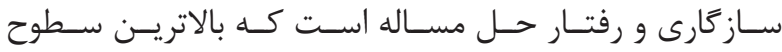

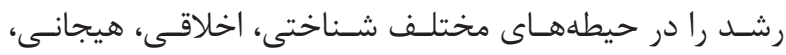

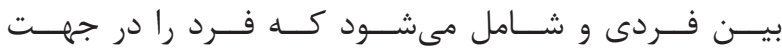

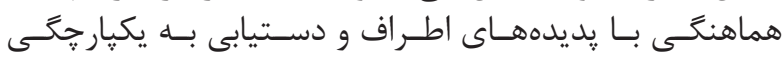

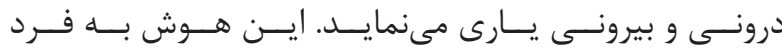

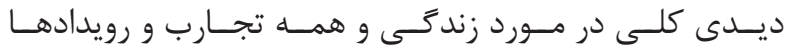

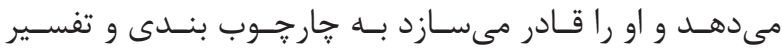

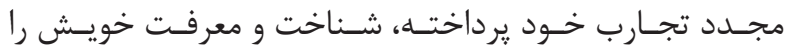

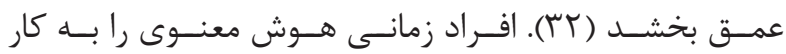

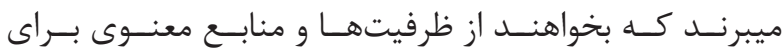

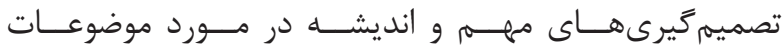

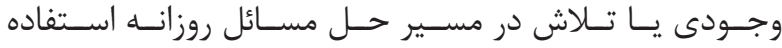

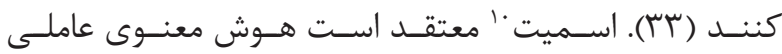

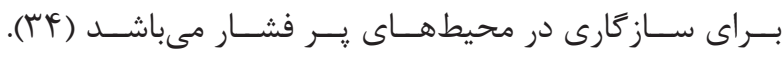

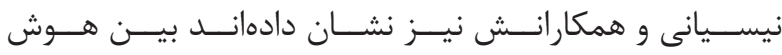

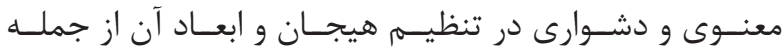

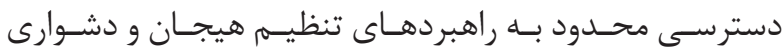

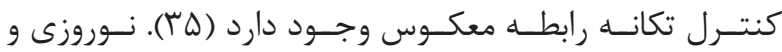

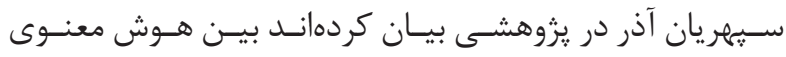

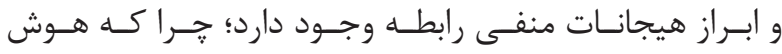

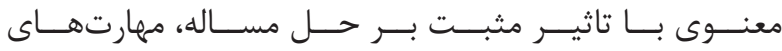

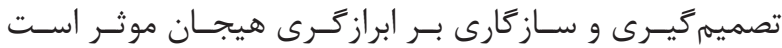

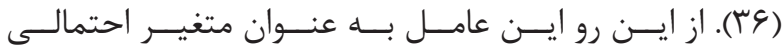

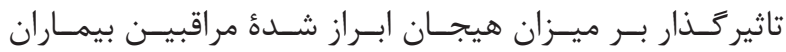

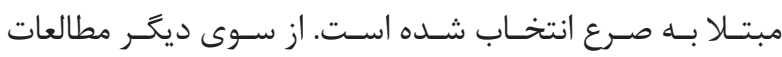

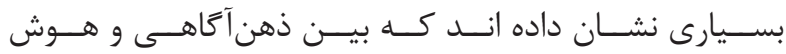

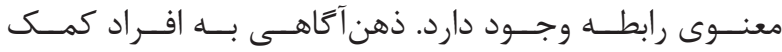

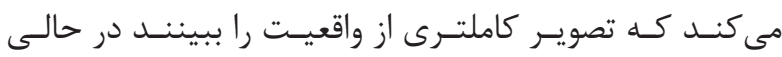

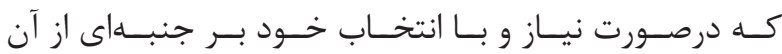

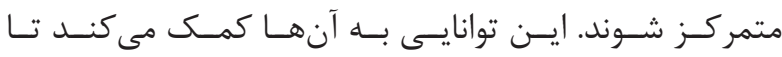

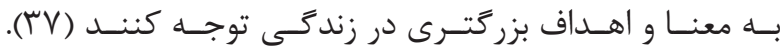

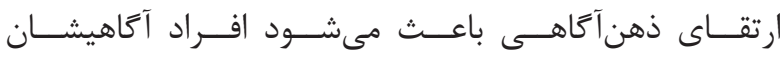

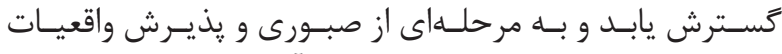

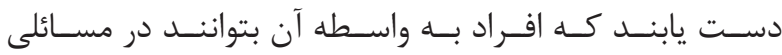

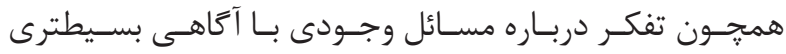

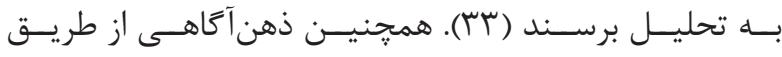

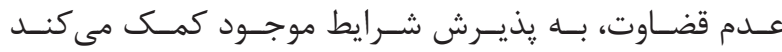

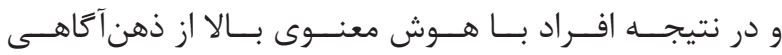

\footnotetext{
${ }^{9}$ Spiritual intelligence

${ }^{11}$ Agarwal \& Mishra 


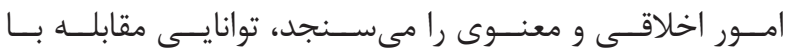

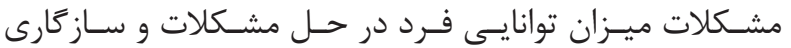

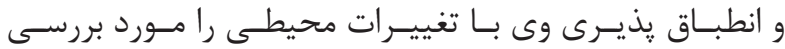

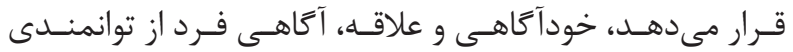

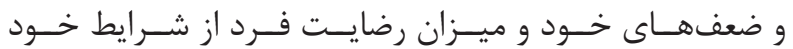

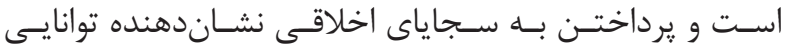

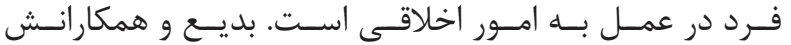

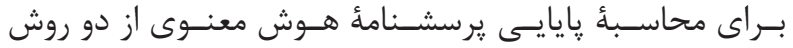

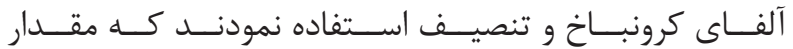

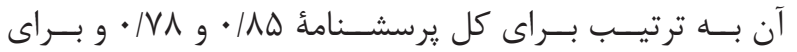

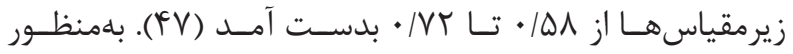

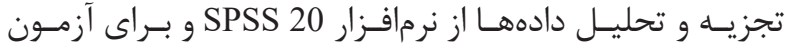

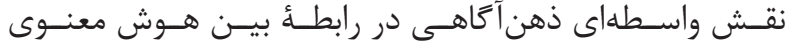

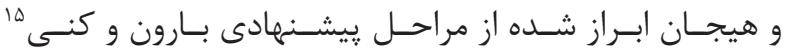

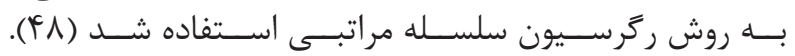

بافتهها

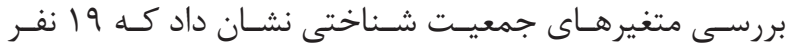

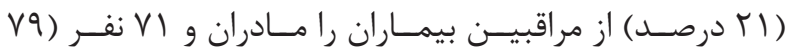

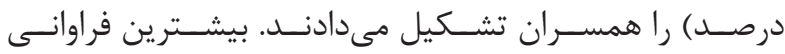

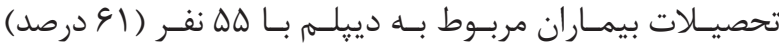

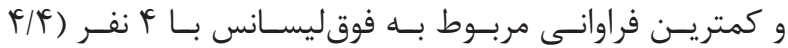

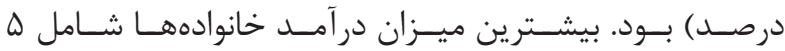

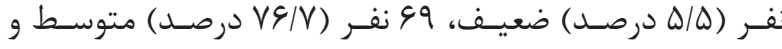

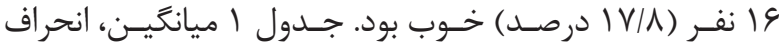

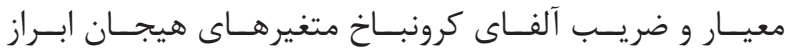

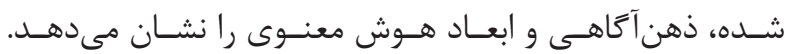

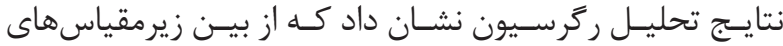

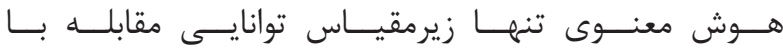

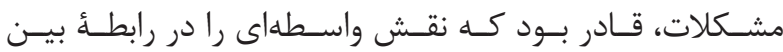

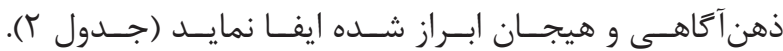

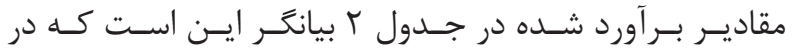

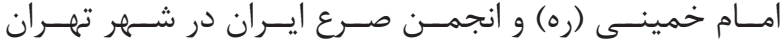

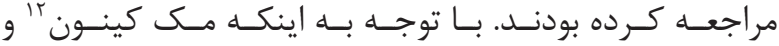

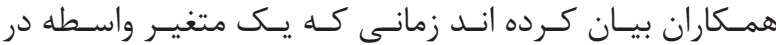

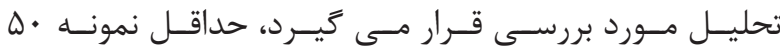

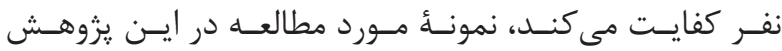

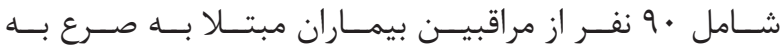

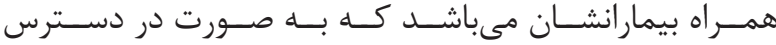

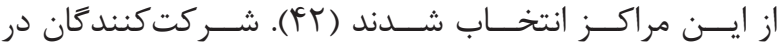

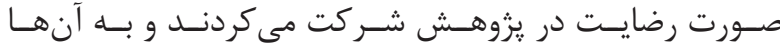

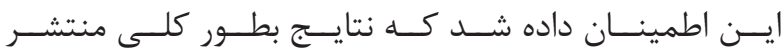

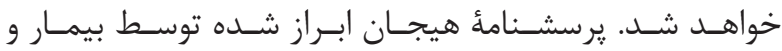

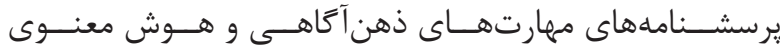

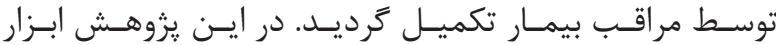

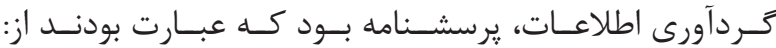

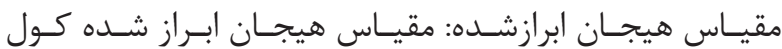

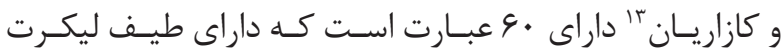

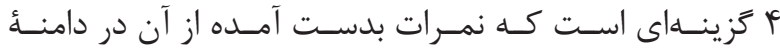

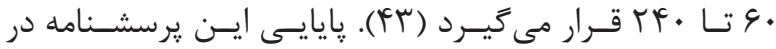

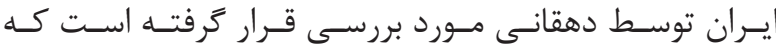

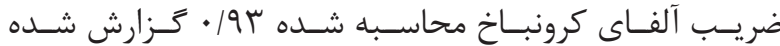

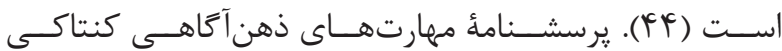

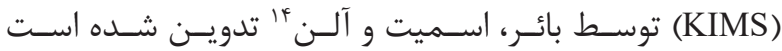

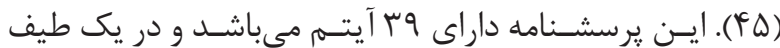

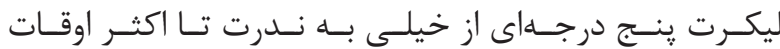

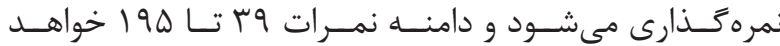

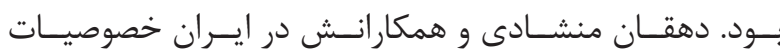

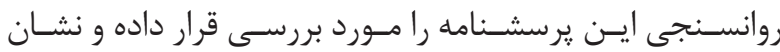

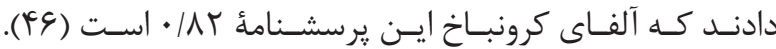

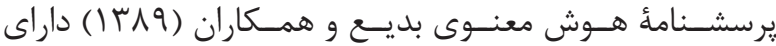

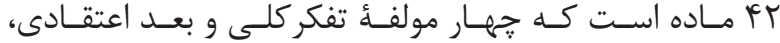

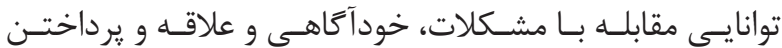

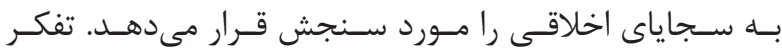

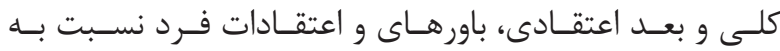

جدول ا- ميانكين، انحراف معيار و آلفاى كرونباخ متغيرهاى برؤهش

\begin{tabular}{|c|c|c|c|c|}
\hline آلفاى كرونباخ در هُوهش حاضر & انحراف معيار & ميانگين & \multicolumn{2}{|c|}{ متغيرها } \\
\hline .199 & $\mathrm{HF} / \mathrm{VQ}$ & IFN/WT & \multicolumn{2}{|c|}{ هيجان ابراز شده } \\
\hline$\cdot|\Lambda|$ & IT/VA & $119 / 90$ & \multicolumn{2}{|c|}{ ذهن آكاهى } \\
\hline.$/ 9 \mathrm{~V}$ & $|0 / f|$ & $r V / \cdot r$ & تفكر كلى و بعد اعتقادى & \multirow{4}{*}{ ابعاد هوش معنوى } \\
\hline$\cdot 119$ & $1 \cdot / 11$ & fe/Af & توانايى مقابله با مشكلات & \\
\hline$\cdot / N \Delta$ & $\boldsymbol{c} / \Lambda \mathrm{r}$ & سT/Fr & خود آكاهى و علاقه & \\
\hline$\cdot \mid \Lambda V$ & $\Delta / 1 T$ & TI/ & هرداختي به سجاياى اخلاقى & \\
\hline
\end{tabular}

${ }^{12}$ MacKinnon

${ }^{13}$ Cole \& Kazarian

${ }^{14}$ Baer, Smith \& Allen

${ }^{15}$ Baron \& Kenny 
جدول r- نتايج تحليل رَّرسيون سلسله مراتبى هيجان ابراز شده براساس مهارتهاى ذهنآكاهى و زيرمقياس توانايى مقابله با مشكلات از هوش معنوى

\begin{tabular}{|c|c|c|c|c|c|c|c|c|}
\hline P-value & $\mathbf{F}$ & $\mathbf{R}^{2}$ & $\mathbf{R}$ & P-value & بتا & ضريب B & متغيرهاى بيشبين & مر حله \\
\hline$\cdot 1 \cdot \cdot 1$ & $19 / \cdot 1$ & $\cdot 1 / \theta$ & . & $\cdot 1 \cdot \cdot 1$ &.$- / 1 \% q$ & $-1 / \cdot 9$ & ذهن آكاهى & 1 \\
\hline \multirow{2}{*}{$\cdot 1 \cdot \cdot 1$} & \multirow{2}{*}{ II/Ar } & \multirow{2}{*}{$\cdot|r|$} & \multirow{2}{*}{.149} & $.1 \cdot 9$ &.$- / r \Lambda$ & $-\cdot / V q$ & ذهن آتاهى & \multirow{2}{*}{$r$} \\
\hline & & & & $\cdot 1 \cdot 1 r$ & $-\cdot / T V$ & $-.19 r$ & توانايى مقابله با مشكلات & \\
\hline
\end{tabular}

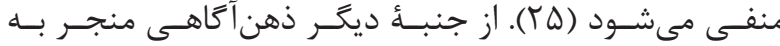

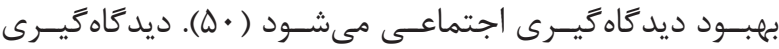

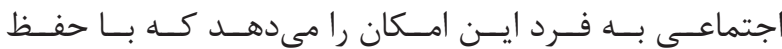

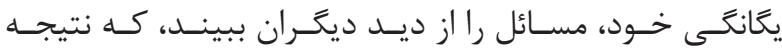

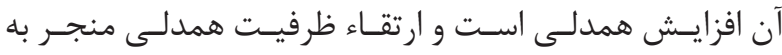

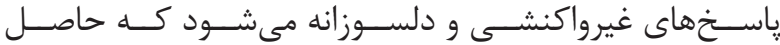

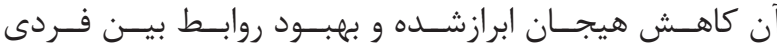

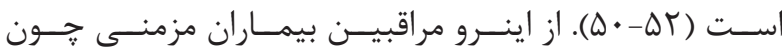

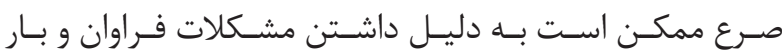

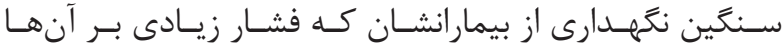

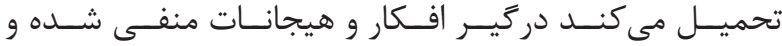

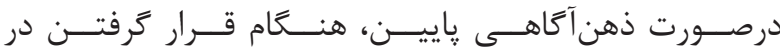

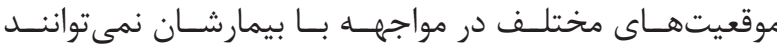

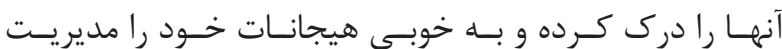

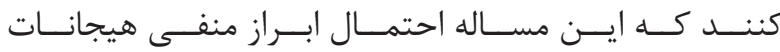

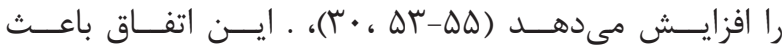

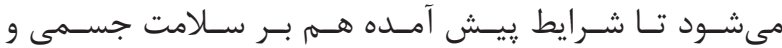

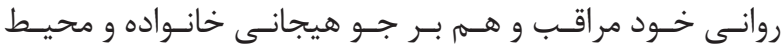

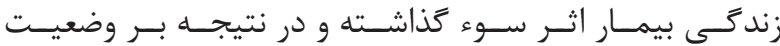

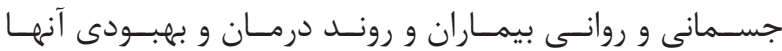

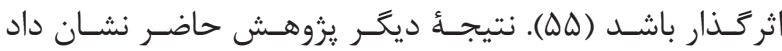

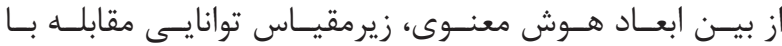

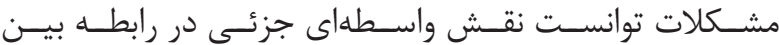

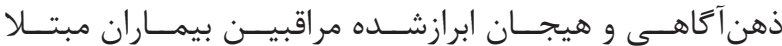

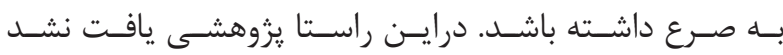

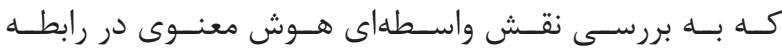

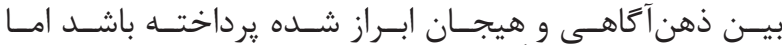

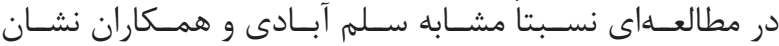

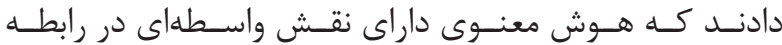

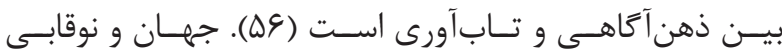

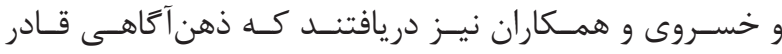

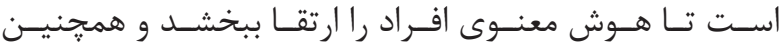

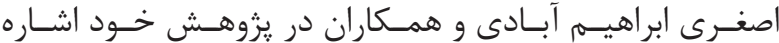

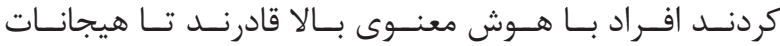

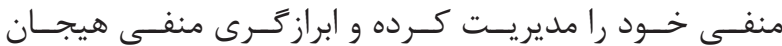

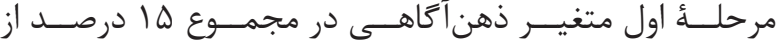

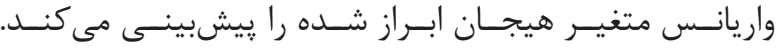

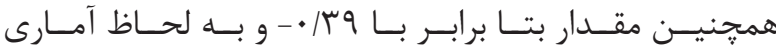

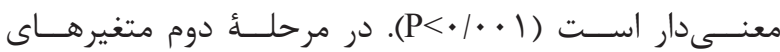

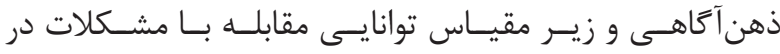

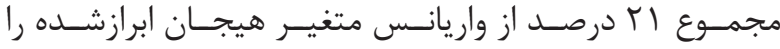

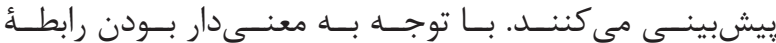

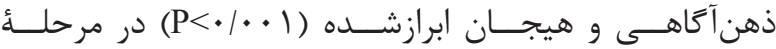

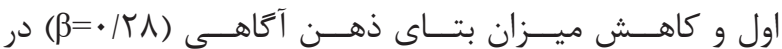

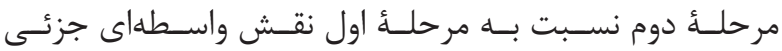

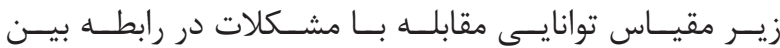

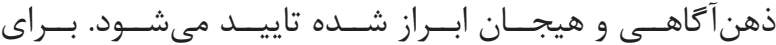

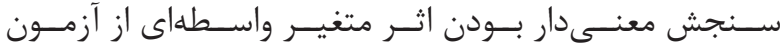

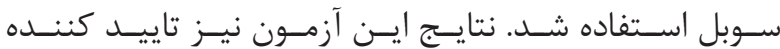

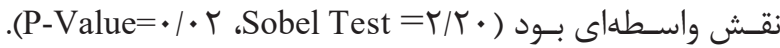

بحث و نتيجه كَيرى

نتايـج يروهـش حاضـر نشـان داد بيـن ذهن آكَاهـى و هيجــان

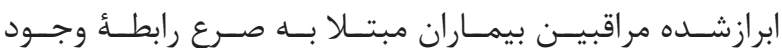

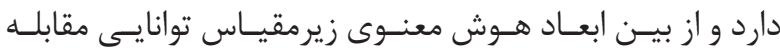

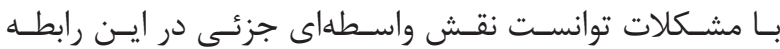

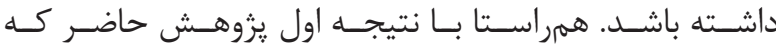

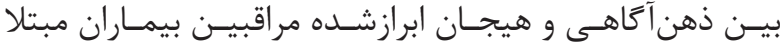

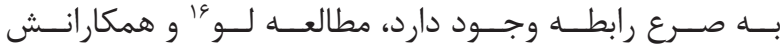

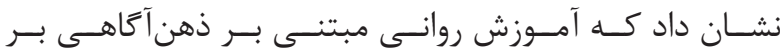

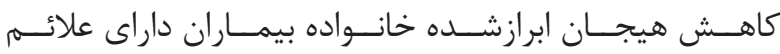

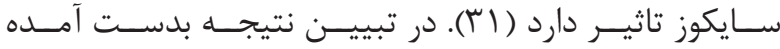

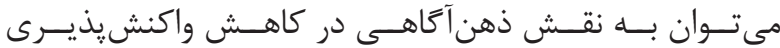

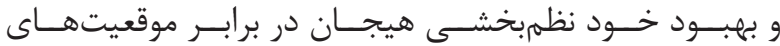

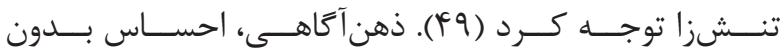

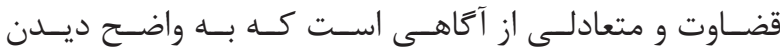

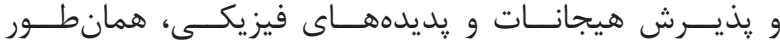

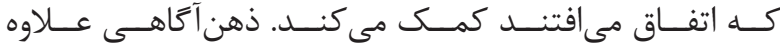

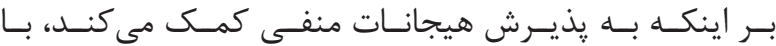

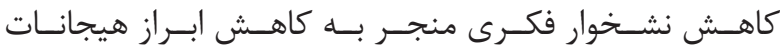




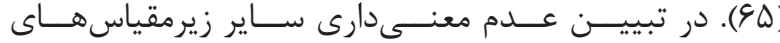

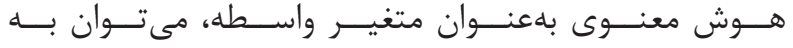

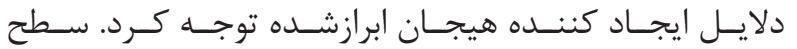

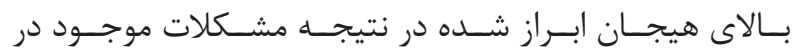

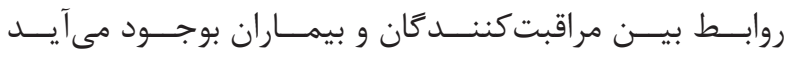

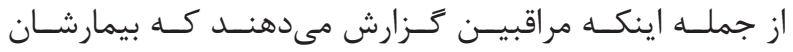

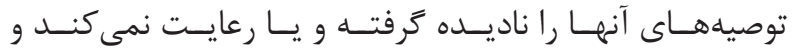

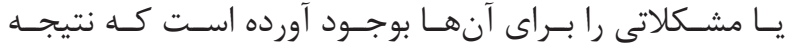

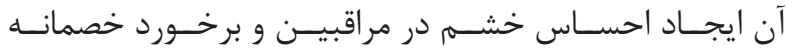

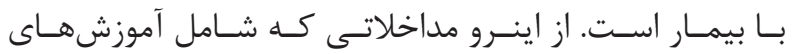

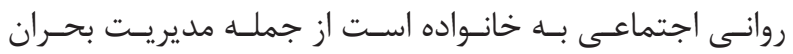

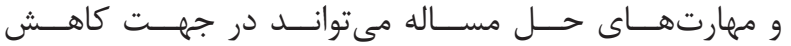

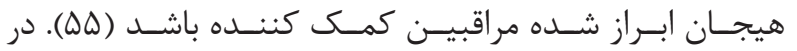

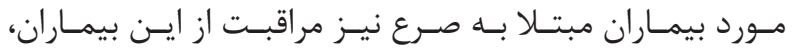

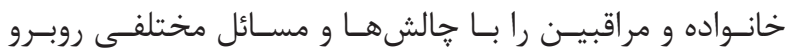

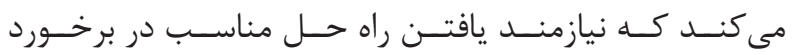

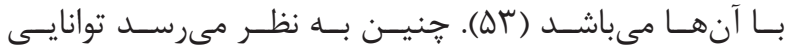

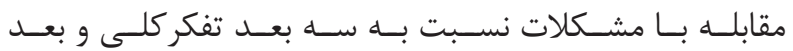

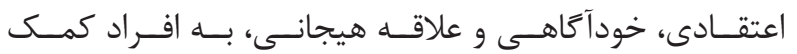

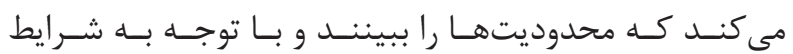

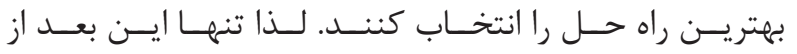

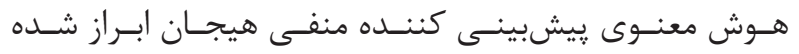

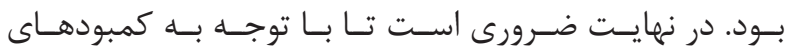

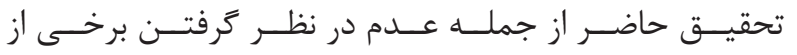

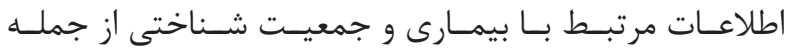

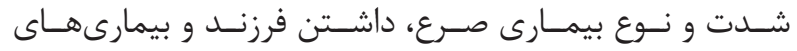

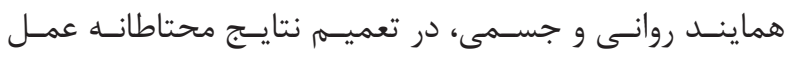

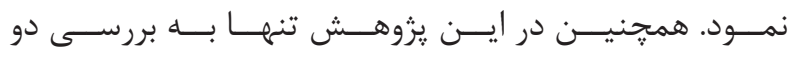

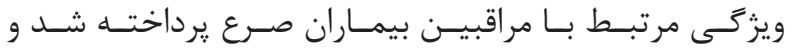

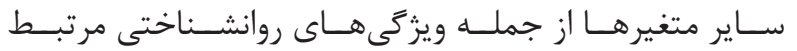

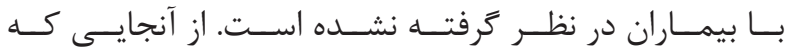

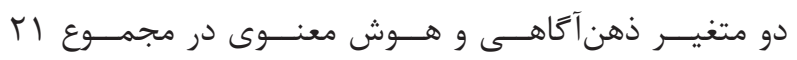

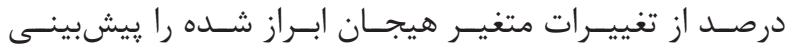

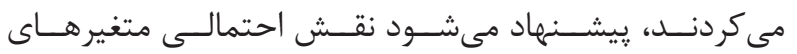

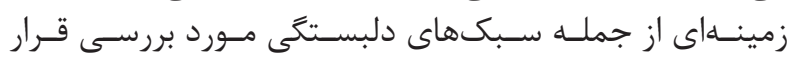

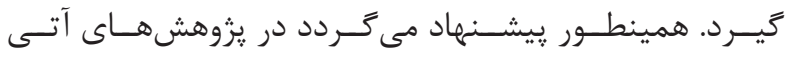

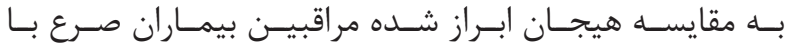

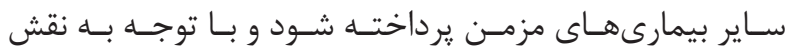

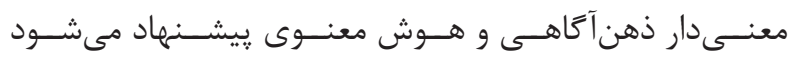

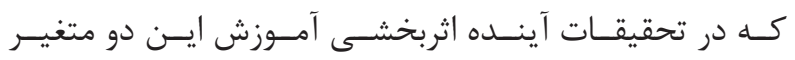

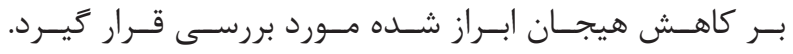

1-Sirven JI. Epilepsy: a spectrum disorder. Cold Spring Harbor perspectives in medicine. 2015; 5(9): a022848.

2- Nonato DT, Vasconcelos SM, Mota MR, de Barros Silva PG, Cunha AP, Ricardo NM, Pereira MG, Assreuy AM, Chaves EM. The anticonvulsant effect of a polysaccharide-rich extract from Genipa ${ }^{17}$ Elkins \& Cavendish

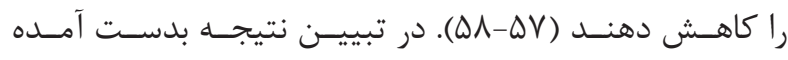

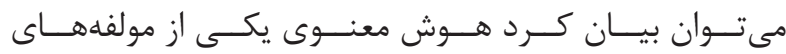

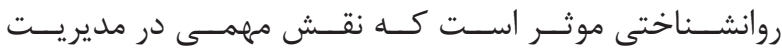

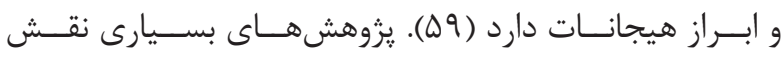

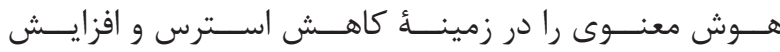

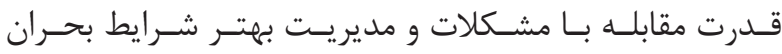

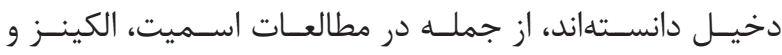

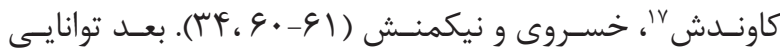

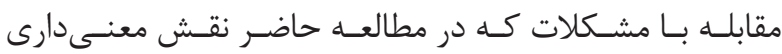

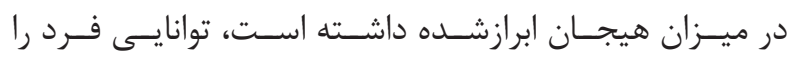

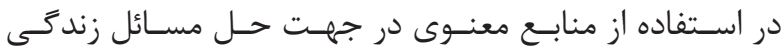

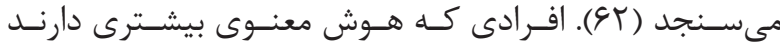

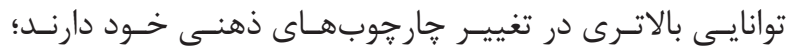

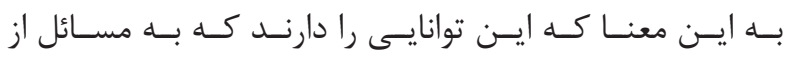

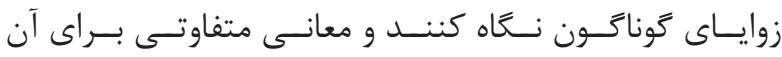

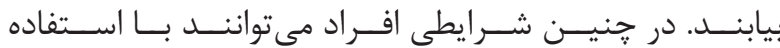

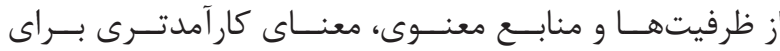

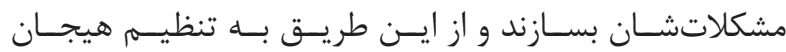

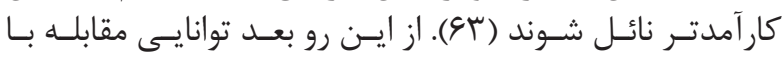

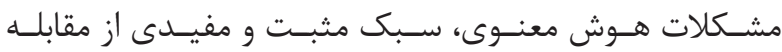

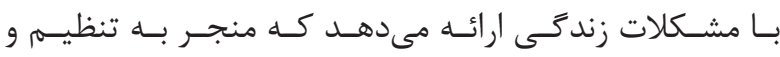

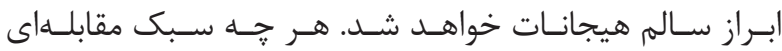

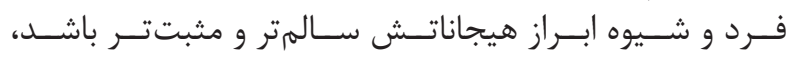

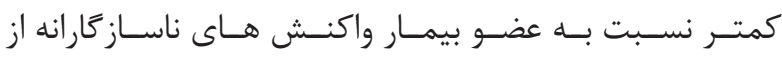

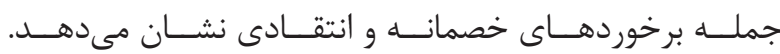

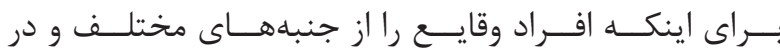

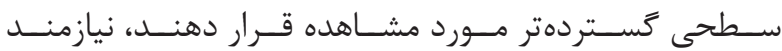

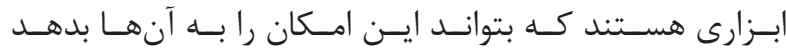

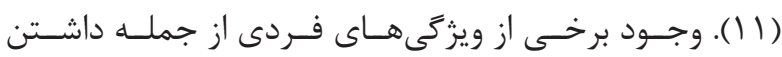

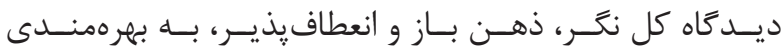

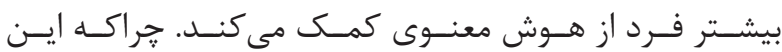

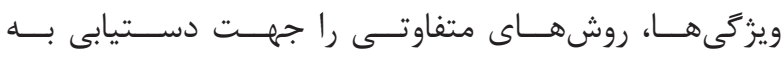

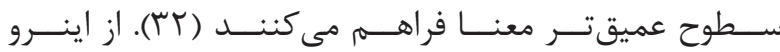

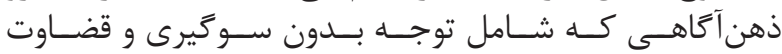

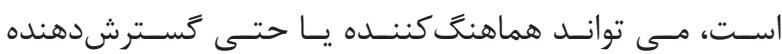

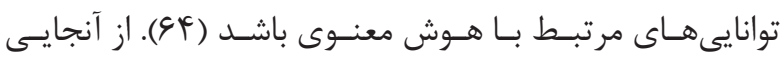

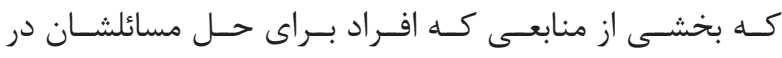

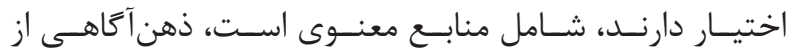

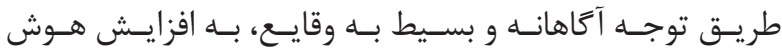

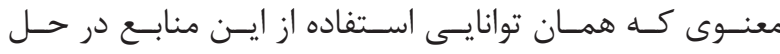

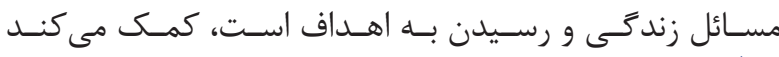
منابع americana leaves is mediated by GABA receptor. Biomedicine \& Pharmacotherapy. 2018; 101: 181-7.

3- Chua KC, Chandran V, Acharya R, Lim CM. Automatic identification of epilepsy by HOS and power spectrum parameters using EEG signals: A comparative study. In2008 30th Annual International 
Conference of the IEEE Engineering in Medicine and Biology Society 2008 (pp. 3824-3827). IEEE.

4- Magalov SI, Hasanov NF, Azizova NX, Novruzov AN, Mustafayev ZB, Kazimov SA, et al. The prevalence of epilepsy in the Nakhichevan Autonomous Republic of Azerbaijan. CNS Neurol Disord Drug Targets. 2012; 11(2): 102-9.

5- Sayehmiri K, Tavan H, Sayehmire F, Mohamadi I. Prevalence of Epilepsy in Iran Using Meta-Analysis and Systematic Review. J Adv Med Biomed Res. 2015; 23 (97): 112-121.

6- Banerjee PN, Filippi D, Hauser WA. The descriptive epidemiology of epilepsy-a review. Epilepsy research. 2009; 85(1): 31-45.

7- O'Dell C, Wheless JW, Cloyd J. The personal and financial impact of repetitive or prolonged seizures on the patient and family. Journal of child neurology. 2007; 22(5_suppl): 61S-70S.

8- de Boer HM. Epilepsy stigma: moving from a global problem to global solutions. Seizure. 2010;19(10):630-6.

9- Mahrer-Imhof R, Jaggi S, Bonomo A, Hediger H, Eggenschwiler P, Krämer G, Oberholzer E. Quality of life in adult patients with epilepsy and their family members. Seizure. 2013; 22(2): 128-35.

10- Lai ST, Tan WY, Wo MC, Lim KS, Ahmad SB, Tan CT. Burden in caregivers of adults with epilepsy in Asian families. Seizure. 2019; 71: 132-9.

11- Wasserman S, Weisman A, Suro G. Nonreligious coping and religious coping as predictors of expressed emotion in relatives of patients with schizophrenia. Mental health, religion \& culture. 2013; 16(1): 16-30.

12- Lobban F, Barrowclough C, Jones S. Does Expressed Emotion need to be understood within a more systemic framework? An examination of discrepancies in appraisals between patients diagnosed with schizophrenia and their relatives. Soc Psychiatry Psychiatr Epidemiol. 2006; 41(1): 50-5.

13- Bressi C, Cornaggia CM, Beghi M, Porcellana M, Iandoli II, Invernizzi G. Epilepsy and family expressed emotion: Results of a prospective study. Seizure. 2007; 16(5): 417-23.

14- Khanipour H, Akbari M. The Relationship between Religious Coping Styles, ShameProneness, Guilt-Proneness and Expressed Emotion among Family Members of Individuals with Chronic Mental Disorder. Journal of Family Research. 2019; 15(57): 89-104. (Persian)
15- Van Noppen B, Steketee G. Testing a conceptual model of patient and family predictors of obsessive compulsive disorder (OCD) symptoms. Behav Res Ther. 2009; 47(1): 18-25.

16- Renshaw KD, Chambless DL, Steketee G. Perceived criticism predicts severity of anxiety symptoms after behavioral treatment in patients with obsessive-compulsive disorder and panic disorder with agoraphobia. J Clin Psychol. 2003; 59(4): 411-21.

17- Hooley JM, Parker HA. Measuring expressed emotion: an evaluation of the shortcuts. J Fam Psychol. 2006 ; 20(3): 386-96.

18- Peris TS, Miklowitz DJ. Parental Expressed Emotion and Youth Psychopathology: New Directions for an Old Construct. Child Psychiatry Hum Dev. 2015; 46(6): 863-73.

19- Hooley JM. Expressed emotion and relapse of psychopathology. Annu. Rev. Clin. Psychol.. 2007; 3: 329-52.

20- Sazvar S A, Nouri R, Saei R, Hatami M. Impact of acceptance and commitment-based psychoeducation on the adjustment of expressed emotion in families of patients with bipolardisorder. Feyz. 2017;21 (3):265-271.

21- Eisner LR, Johnson SL. An acceptancebased psychoeducation intervention to reduce expressed emotion in relatives of bipolar patients. Behavior therapy. 2008; 39(4): 375-85.

22- Kabat-Zinn J. Mindfulness-Based Interventions in Context: Past, Present, and Future. Clinical Psychology: Science and Practice. 2003; 10(2): 144-56.

23- Baer RA, Smith GT, Allen KB. Assessment of mindfulness by self-report: the Kentucky inventory of mindfulness skills. Assessment. 2004; 11(3): 191-206.

24- Ryan R, Brown K. Why We Don't Need SelfEsteem: On Fundamental Needs, Contingent Love, and Mindfulness. Psychological Inquiry. 2003; 14.

25- Brown KW, Ryan RM, Creswell JD. Addressing Fundamental Questions About Mindfulness. Psychological Inquiry. 2007; 18(4): 272-81.

26- Praissman S. Mindfulness-based stress reduction: a literature review and clinician's guide. J Am Acad Nurse Pract. 2008; 20(4): 212-6.

27- Ndubisi N. Mindfulness, reliability, preemptive conflict handling, customer orientation and outcomes in Malaysia's healthcare sector. Journal of Business Research. 2012; 65: 537-46. 
28- Babaei Nadinluye K, Mikaeli Manee F, Pezeshki $\mathrm{H}$, Bafande $\mathrm{H}$, Abdi $\mathrm{H}$. Relationship between Mindfulness and Meta-Emotion on Predicting Emotional Adjustment of Novice Nurses. Iran Journal of Nursing. 2017; 30(105): 11-22. (Persian)

29-Kor PP, Liu JY, Chien WT. Effects on stress reduction of a modified mindfulness-based cognitive therapy for family caregivers of those with dementia: study protocol for a randomized controlled trial. Trials. 2019;20(1):303.

30- Singh NN, Lancioni GE, Karazsia BT, Chan J, Winton AS. Effectiveness of caregiver training in mindfulness-based positive behavior support (MBPBS) vs. training-as-usual (TAU): a randomized controlled trial. Frontiers in psychology. 2016; 7: 1549

31- Lo HH, Ho WC, Lau EN, Lo CW, Mak WW, Ng SM, Wong SY, Wong JO, Lui SS, Lo CS, Lin EC. A Brief Mindfulness-Based Family Psychoeducation Intervention for Chinese Young Adults With First Episode Psychosis: A Study Protocol. Frontiers in psychology. 2019; 10: 516.

32- Ghobari Banab, B., Salimi M., Soleymani L, Noori Moghadam S. (2007). Spiritual intelligence. Innovate religious ideas; 3 (10): 125-147. (Persian)

33- Jahan F, Noghabi R. The Effect of Mindfulness Training on Moral Intelligence, Spiritual Intelligence and Wisdom in the Elderly. mejds. 2019; 9: 17 (Persian).

34- Smith S. Exploring the Interaction of Trauma and Spirituality. Traumatology.2004.

35- Mahdavi Neysiani Z, Asadi A, Asgari M, Ghale' Noei F. Relationship of religious orientation and spiritual intelligence with emotional selfregulation in women subject to violence. J Res Relig Health. 2019;5(2):101-114. (Persian)

36- Norouzi M, Sepehrian Azar F. Comparing spiritual intelligence and emotional expressiveness in psychosomatic patients. J Research Health. 2017; 7 (2) :745-753. (Persian)

37- Gieseke AR. The relationship between spiritual intelligence, mindfulness, and transformational leadership among public higher education leaders (Doctoral dissertation, Northeastern University).

38- Lazaridou A, Pentaris P. Mindfulness and spirituality: therapeutic perspectives. Person-Centered \& Experiential Psychotherapies. 2016; 15(3): 235-44.

39- Agarwal S, Mishra PC. Relationship between mindfulness and spiritual intelligence among bank employees. Indian Journal of Positive Psychology. 2016; 7(3): 356.

40- Tabarsa G, Moeini M. the Relationship between Dimensions of Spiritual Intelligence and Reducing Job Burnout. ORMR. 2015; 4 (4): 109-135. (Persian)

41- Rahmani Anaraki H, Mahmoodi G R, Rouhi G, Asayesh H, Nasiri H, Rakhshani H. General Health Status of Neurologic Patients' Caregivers and The Related Factors.JRes DevNurs Midw.2013;9(2):49-55.(Persian)

42- MacKinnon DP, Fairchild AJ, Fritz MS. Mediation analysis. Annu. Rev. Psychol. 2007 Jan 10;58:593-614.

43- Cole JD, Kazarian SS. The level of expressed emotion scale: a new measure of expressed emotion. Journal of Clinical psychology. 1988; 44(3): 392-7.

$\begin{array}{lcccr}\text { 44- } & \text { Dehghani } & \text { Sh. } & \text { Expressed } & \text { Emotion } \\ \text { and } & \text { Sexuality. } & \text { Fundamentals } & \text { of } & \text { Mental } \\ \text { Health } & 2003 ; 6(19): 84-91 . & & \text { (Persian) }\end{array}$

45- Baer RA, Smith GT, Allen KB. Assessment of mindfulness by self-report: The Kentucky Inventory of Mindfulness Skills. Assessment. 2004; 11(3): 191-206.

46- Dehghan Manshadi Z, Taghavi MR, Dehghan Manshadi M. Psychometric characteristics of the Kentucky inventory of mindfulness skills. Journal of Clinical Psychology (Andishe va Raftar). 2012; 7(25): 27-36. (Persian)

47- Badie A, Savari E, Bagheri Dashtbozorg N, Latifiazadegan V. Development and reliability and validity of the spiritual intelligence scale. First National Psychology Conference Payamnour University, Tabriz.2010. (Persian)

48- Baron RM, Kenny DA. The moderatormediator variable distinction in social psychological research: Conceptual, strategic, and statistical considerations. Journal of personality and social psychology. 1986 Dec; 51(6): 1173.

49- Bränström R, Duncan LG, Moskowitz JT. The association between dispositional mindfulness, psychological well-being, and perceived health in a Swedish population-based sample. $\mathrm{Br} J$ Health Psychol. 2011; 16(Pt 2): 300-16.

50- Shokri A, Kazemi R, Narimani M, Taklavi S. Comparison of the Effectiveness of Mother's Mindfulness-based Cognitive Therapy and Cognitive Emotion Regulation Training on Externalizing Disorder and Self-efficacy of Aggressive Children. Practice in Clinical Psychology. 2020; 8(2): 85-98.

51- Saidi, S, Ahmadian, H, saffarinia, M. The Model 
Of Relationship Between Social Perspective Taking And Self-Control With Pro-social Personality Through Mediator Role Emotional Empathy. Social Cognition, 2019; 8(2): 80-90. (Persian)

52- Smith EL, Jones FW, Holttum S, Griffiths K. The process of engaging in mindfulness-based cognitive therapy as a partnership: a grounded theory study. Mindfulness. 2015; 6(3): 455-66.

53- Heidari M, Etemadi far S, Masoudi R, Kheyri S, Jivad N. The effect of family-centered care on the family caregivers' burden of patients with epilepsy . Journal of Clinical Nursing and Midwifery. 2018; 7(2): 146-157.

54- Wijma EM, Veerbeek MA, Prins M, Pot AM, Willemse BM. A virtual reality intervention to improve the understanding and empathy for people with dementia in informal caregivers: results of a pilot study. Aging \& mental health. 2018; 22(9): 1121-9.

55- Amaresha AC, Venkatasubramanian G. Expressed emotion in schizophrenia: an overview. Indian journal of psychological medicine. 2012; 34(1): 12-20.

56- Salmabadi M, Khamesan A, Usefynezhad A, Sheikhipoor M. The Mediating Role of Spiritual Intelligent in Relationship of Mindfulness and Resilience. Health, Spirituality and Medical Ethics. 2016 Sep 10;3(3):18-24. (Persian)

57- Khosravi M, Tahmasebipour N, Jarrareh J. The Effectiveness of Mindfulness Group Training on Enhancing Students' Spiritual Intelligence. Second International Conference on Psychology, Counseling and Education. Mashhad, Shandiz Higher Education Institution. 2018. (Persian)

58- Asghari Ebrahimabad M J, Moshirian Farahi M
M, Najmi M, Ghafari Moslemi H et al . Prediction of Difficulty in Emotional Adjustment Based on Spiritual Intelligence among Criminals at Mashhad Prison. JRH. 2015; 3(1): 39-48. (Persian)

59- Mahdavi Neysiani Z, Asadi A, Asgari M, Ghale' Noei F. Relationship of religious orientation and spiritual intelligence with emotional selfregulation in women subject to violence. J Res Relig Health.2019;5(2):101-114. (Persian)

60- Elkins M, Cavendish R. Developing a plan for pediatric spiritual care. Holistic Nursing Practice. 2004 Jul 1; 18(4): 179-84.

61- Khosravi M, Nikmanesh Z. Relationship of spiritual intelligence with resilience and perceived stress. Iranian journal of psychiatry and behavioral sciences. 2014; 8(4): 52 .

62- Zarei Matin H, KheirAndish M, Jahani H. ConceptualizationofSpiritual IntelligenceinOrganization (Case Study in Labbafi Nejad Hospital of Tehran). Management Researches. 2011; 4(12): 71-94.(Persian)

63- Mohammadi Kanjani B, Mazaheri MA, Heidari M, Moradi A. The Relationship of the Spiritual Intelligence and Adherence to Religious Beliefs with the Cognitive Emotion Regulation Among Pregnant Women. Ravanshenasi Va Din. 2017; 10(2): 125-142. (Persian)

64- Skrzypińska K. Does spiritual intelligence (SI) exist? A theoretical investigation of a tool useful for finding the meaning of life. Journal of Religion and Health. 2020; 1-7.

65- Zamiri, S, Azizi, S, Shakeri, A, HasanDoost, Z, Mohammaddost, M, Yosefi, H, Moslem, A, Akaberi, A. Predicting Self-Efficacy of Students Based on Spiritual Intelligence. Journal of Sabzevar University of Medical Sciences, 2016; 23(2): 196-203. (Persian) 